\title{
Resistência de helmintos gastrintestinais de bovinos a anti-helmínticos no Planalto Catarinense
}

\author{
Anthelmintics resistance of bovine gastrointestinal helminths in Santa Catarina Plateau
}

\author{
Antonio Pereira de Souza ${ }^{I^{*}}$ César Itaqui Ramos ${ }^{\mathrm{II}}$ Valdomiro Bellato ${ }^{\mathrm{I}}$ Amélia Aparecida Sartor $^{\mathrm{I}}$ \\ Crisciane Aparecida Schelbauer ${ }^{\mathrm{II}}$
}

\section{RESUMO}

Com o objetivo de avaliar focos de resistência de helmintos gastrintestinais parasitos de bovinos, com aptidão para corte, à ivermectina, ao fosfato de levamisole e ao sulfóxido de albendazole foram realizadas avaliações em 39 propriedades localizadas no Planalto Catarinense. Em cada uma foram coletadas, em média, 60 amostras de fezes de animais, com idade entre sete e 18 meses, para avaliar o número de ovos por grama de fezes (OPG), o cultivo e a identificação de larvas. Foram formados três grupos de animais com média de OPG semelhante. A média de OPG de todos os animais coletados, antes do tratamento, foi considerada como testemunha. Cada grupo recebeu um tratamento diferente por via subcutânea (ivermectina na dose de $0,2 \mathrm{mg} \mathrm{kg}^{-1}$, fosfato de levamisole na dose de $5 \mathrm{mg} \mathrm{kg}^{-1}$ e sulfóxido de albendazole na dose de $2,5 \mathrm{mg} \mathrm{kg}^{-1}$ ). Sete dias após foram repetidas as avaliações por grupo. Considerou-se resistência quando a eficácia da droga foi menor que 95\% e o limite inferior do intervalo de confiança IC $95 \%$ menor que $90 \%$. Entre as propriedades avaliadas, 82,1\% apresentaram animais com helmintos resistentes à ivermectina, 15,4\% ao fosfato de levamisole e 7,8\% ao sulfóxido de albendazole. Em apenas 10,3\% das propriedades, a eficácia de todos os anti-helmínticos foi superior a 95\%. Com base no diagnóstico genérico, verificou-se que os gêneros predominantes resistentes à ivermectina foram Cooperia spp e Haemonchus spp, ao fosfato de levamisole, Ostertagia spp, Cooperia spp e Trichostrongylus spp, e ao sulfóxido de albendazole, Cooperia spp.

Palavras-chave: Trichostrongylidae, sulfóxido albendazole, fosfato levamisole, ivermectina.

\section{ABSTRACT}

In order to estimate possible focus of resistance of bovine gastrointestinal helminths to ivermectin, levamisole phosphate and albendazole sulphoxide, 39 properties in the

\begin{abstract}
Santa Catarina Plateau were evaluated. In each location a mean number of 60 samples of feces was collected from animals between seven and 18 months of age, for the evaluation of the number of eggs per gram of feces (EPG), for culture and for larvae identification. Three groups with similar EPG mean were comprised, with the mean EPG being considered the group control. Animal from each group received a different subcutaneous treatment: a) $0.2 \mathrm{mg} \mathrm{kg}^{-1}$ ivermectin, b) $5 \mathrm{mg} \mathrm{kg} \mathrm{kg}^{-1}$ levamisole phosphate, and c) $2.5 \mathrm{mg} \mathrm{kg}^{-1}$ albendazole sulphoxide). The fecal evaluation, per group, was repeated seven days later. Resistance was considered when the efficacy of the drug was lesser than 95\% and when the inferior limit of the confidence interval CI95\% was lesser than $90 \%$. From the properties examined, $82.1 \%$ presented animals with helminths resistant to ivermectin, $15.4 \%$ to levamisole phosphate, and $7.8 \%$ to albendazole sulphoxide. In only $10.3 \%$ of the properties the efficacy of all the anthelmintics was superior to 95\%. Based on the generic diagnosis, through the larvae identification, Cooperia spp and Haemonchus spp were the prevailing resistant genus to ivermectin, Ostertagia spp, Cooperia spp and Trichostrongylus spp were associated with resistance to levamisole phosphate, and Cooperia spp. to albendazole sulphoxide.
\end{abstract}

Key words: Trichostrongylidae, albendazole sulphoxide, levamisole phosphate, ivermectin.

\section{INTRODUÇÃO}

De acordo com o levantamento feito durante os anos de 2002-2003, o rebanho bovino catarinense era composto por 3.226.293 cabeças, das quais $14,73 \%$ estavam localizadas no Planalto Catarinense (INSTITUTO CEPA/SC, 2005). Os índices de produtividade são considerados baixos, ocasionados

\footnotetext{
IDepartamento de Medicina Veterinária Preventiva e Tecnologia, Centro de Ciências Agroveterinárias (CAV), Universidade do Estado de Santa Catarina (UDESC), Lages, SC, Brasil. E-mail: a2aps@cav.udesc.br. *Autor para correspondência.

"Empresa de Pesquisa Agropecuária e Extensão Rural de Santa Catarina S. A. (EPAGRI), Lages, SC, Brasil.

IIICurso de Medicina Veterinária, UDESC, Lages, SC, Brasil.
} 
principalmente pela flutuação estacional na produção de pastagens e pelas enfermidades parasitárias. As infecções por nematóides têm importância econômica mundial na criação de animais domésticos (PRICHARD, 1994), em função de limitar a produção de leite, reduzir o ganho de peso e a conversão alimentar, além de comprometer o desempenho reprodutivo e o sistema imunológico (COSTA, 2004). SOUZA et al. (1994) verificaram que o controle de helmintos gastrintestinais, de berne e de carrapatos proporcionou, no período de janeiro a agosto, um ganho de peso médio de 53kg, quando comparados a animais não medicados. RAMOS et al. (1984) observaram, no Planalto Catarinense, que animais após o desmame, tratados a cada 45 dias com levamisole, reduziram em um ano e meio o tempo gasto para atingir $380 \mathrm{~kg}$, quando comparado com bovinos não-tratados.

O uso intensivo de anti-helmínticos, subdoses, e diagnósticos incorretos e a falta de rotatividade de bases farmacológicas têm provocado um sério problema sanitário, que é a resistência de nematóides aos fármacos. Este fenômeno é definido como a capacidade hereditária de uma população parasitária de reduzir a sua sensibilidade à ação de uma ou mais drogas (FIEL et al., 2003).

A resistência de helmintos a anti-helmínticos em ovinos e caprinos é freqüente no sistema de produção. Todavia, o mesmo não acontece com helmintos de bovinos, existindo um número menor de relatos. Porém, isso não é indicativo de que os parasitos dessa espécie apresentem uma menor densidade genética para a expressão da resistência, mas sim a menor freqüência de tratamentos a que esta espécie é submetida (PAIVA et al., 2001). No entanto, FIEL et al. (2006) relataram que o problema parece ser diferente em países da Oceania, Europa e América do Sul. Neste último continente, a resistência tem sido relatada no Brasil, na Argentina e mais recentemente no Uruguai.

No Brasil, o primeiro relato em bovinos foi feito por PINHEIRO \& ECHEVARRIA(1990), no Rio Grande do Sul, que verificaram resistência do $\boldsymbol{H}$. contortus ao oxfendazole e ao albendazole. SOUZA et al. (2001), em Santa Catarina, publicaram resultados parciais sobre a resistência de Trichostrongylus spp e Ostertagia spp ao levamisole, Haemonchus spp e Cooperia spp à ivermectina e Cooperia spp ao sulfóxido de albendazole. Mais recentemente, a resistência também foi demonstrada por PAIVA et al. (2001) e COSTA et al. (2004) em São Paulo e por BORGES et al. (2004) e RANGEL et al. (2005) em Minas Gerais.

Com o objetivo de detectar focos de resistência de helmintos gastrintestinais de bovinos a anti-helmínticos à base de ivermectina, fosfato de levamisole e sulfóxido de albendazole foi realizado o presente trabalho.

\section{MATERIAL E MÉTODOS}

Foram coletadas amostras de fezes de bovinos, com aptidão para corte, em 39 propriedades rurais do Estado de Santa Catarina, localizadas no Planalto Catarinense, durante o período de agosto de 2000 a julho 2006. Em cada uma foram coletadas aproximadamente 60 amostras de animais com idade entre sete e 18 meses, para identificação e contagem de ovos por grama de fezes (OPG) pela técnica de GORDON \& WHITLOCK (1939) modificada. A média de OPG nos bovinos de cada propriedade, antes do tratamento, foi considerada controle. Realizou-se o cultivo de larvas pela técnica de ROBERTS \& O'SULLIVAN (1949) e a identificação dos gêneros por meio da chave de KEITH (1953).

Com base nos valores de OPG, os animais foram divididos em três grupos. O grupo 1 foi tratado com ivermectina $\left(0,2 \mathrm{mg} \mathrm{kg}^{-1}\right)$, o grupo 2 com fosfato de levamisole (5mg kg-1) e o grupo 3 com sulfóxido de albendazole $\left(2,5 \mathrm{mg} \mathrm{kg}^{-1}\right)$, todos por via subcutânea. Sete dias após o tratamento foram repetidas as mesmas avaliações por grupo.

Foi aplicado um questionário padrão para obtenção de informações sobre a freqüência e o período de utilização de tratamentos anti-helmínticos, bases químicas e doses empregadas. Foram calculados os percentuais de redução de OPG e os percentuais de participação de cada gênero. Os dados foram tabulados e analisados, considerando-se como resistência quando a eficácia dos anti-helmínticos foi menor que 95\% (COLES et al., 1992) e o intervalo inferior de confiança IC95\% menor que $90 \%$.

\section{RESULTADOS E DISCUSSÃO}

Os resultados da redução de OPG de helmintos gastrintestinais de bovinos pelos antihelmínticos ivermectina, fosfato de levamisole e sulfóxido de albendazole e o número e percentual de propriedades rurais do Planalto Catarinense, agrupados por intervalo de classe, estão expressos na tabela 1.

Observou-se que a ivermectina apresentou eficácia superior a 95\% em apenas 18,0\% das propriedades e eficácia inferior a 14\%, em 5,1\%. O levamisole e o sulfóxido de albendazole apresentaram eficácia superior a 95\% em $84,6 \%$ e $92,1 \%$ das propriedades, respectivamente.

O teste de redução de OPG é um dos mais utilizados em todo o mundo para avaliar a eficácia dos anti-helmínticos ou apontar possíveis focos de resistência, por ser um método simples e de baixo custo. Entretanto, ele apresenta algumas limitações, pois não é capaz de detectar baixos níveis de resistência (MARTIN et al., 1989). Quando se utiliza levamisole, as larvas histotrópicas sensíveis podem sobreviver ao 
Tabela 1- Eficácia da ivermectina, fosfato de levamisole e sulfóxido de albendazole, na redução do número de ovos de helmintos gastrintestinais por grama de fezes, em bovinos de 39 propriedades do Planalto Catarinense.

\begin{tabular}{|c|c|c|c|c|c|c|}
\hline \multirow{2}{*}{ Intervalo de eficácia (\%) } & \multicolumn{2}{|c|}{------Ivermectina------ } & \multicolumn{2}{|c|}{----Fosfato de levamisole---- } & \multicolumn{2}{|c|}{----Sulfóxido de albendazole---- } \\
\hline & $\mathrm{N}^{\mathrm{o}}$ & $\%$ & $\mathrm{~N}^{\mathrm{o}}$ & $\%$ & $\mathrm{~N}^{\mathrm{o}}$ & $\%$ \\
\hline $95-100$ & 7 & 18,0 & 33 & 84,6 & 35 & 92,1 \\
\hline $75-94$ & 10 & 25,6 & 5 & 12,8 & 2 & 5,3 \\
\hline $60-74$ & 7 & 18,0 & 0 & 0,0 & 1 & 2,6 \\
\hline $45-59$ & 7 & 18,0 & 1 & 2,6 & 0 & 0,0 \\
\hline $30-44$ & 3 & 8,0 & 0 & 0,0 & 0 & 0,0 \\
\hline $15-29$ & 3 & 8,0 & 0 & 0,0 & 0 & 0,0 \\
\hline $0-14$ & 2 & 5,1 & 0 & 0,0 & 0 & 0,0 \\
\hline Total & 39 & 100,0 & 39 & 0,0 & 38 & 100,0 \\
\hline
\end{tabular}

$\mathrm{N}^{\mathrm{o}}=$ número de propriedades.

tratamento e, quando as contagens de OPG são realizadas 10 a 14 dias após a medicação, elas podem ter se desenvolvido dando um resultado falso positivo de resistência. Por esse motivo, GRIMSHAW et al. (1996) recomendam fazer a contagem de OPG sete dias após o tratamento. Todavia, algumas espécies resistentes às lactonas macrocíclicas podem sofrer uma inibição temporária da postura, dando um resultado negativo de resistência (JACKSON, 1993).

Verificou-se que das 39 propriedades, em apenas quatro (10,3\%), a redução de OPG, nos bovinos, foi superior a 95\% para os três anti-helmínticos. Utilizando os critérios citados por COLES et al. (1992), ocorreu resistência de helmintos à ivermectina em $82,1 \%$ das propriedades, das quais 5,1\% também apresentavam resistência ao fosfato de levamisole; $2,6 \%$ ao sulfóxido de albendazole e 2,6\% aos três medicamentos. Resistência somente ao fosfato de levamisole foi 5,1\% (Tabela 2).

A maioria dos proprietários não tem informações precisas sobre a freqüência de tratamentos e dos anti-helmínticos utilizados, contudo, respeitam as doses recomendadas pelos fabricantes. A base química utilizada está geralmente na dependência de preço, propaganda e recomendação de vendedores.
No entanto, sempre que houve redução de eficácia, havia histórico de uso anterior do mesmo princípio ativo ou do mesmo grupo químico. Os percentuais de resistência obtidos neste trabalho são superiores aos encontrados por ECHEVARRIA \& PINHEIRO (1999), que avaliaram a eficiência dos mesmos princípios ativos em bovinos de 56 municípios do Rio Grande do Sul e constataram que $20 \%$, 6\%, e $4 \%$ das propriedades apresentavam resistência (reduções de OPG abaixo de $90 \%$ ) respectivamente à ivermectina, levamisole e albendazole. Estas diferenças estão relacionadas, provavelmente, ao manejo e à época de realização dos experimentos.

PAIVA et al. (2001), em São Paulo, demonstraram a presença de resistência às espécies H. placei e C. punctata à ivermectina e mais recentemente COSTA et al. (2004) relataram resistência do Haemonchus e Cooperia à mesma molécula. BORGES et al. (2004) e RANGEL et al. (2005), em Minas Gerais, relataram, com base em teste de redução do número de OPG, provável resistência de Haemonchus e Cooperia à ivermectina e doramectina.

Na tabela 3 estão registrados o número e os percentuais de propriedades com helmintos resistentes aos anti-helmínticos e os percentuais por gênero.

Tabela 2 - Percentual de propriedades com criação de bovinos com helmintos resistentes a um ou mais anti-helmínticos (ivermectina, fosfato de levamisole e sulfóxido de albendazole) no Planalto Cat ari nense.

\begin{tabular}{lcc}
\hline Anti-helmïntico & No de propriedades & \% \\
\hline Ivermectina & 28 & 71,8 \\
Fosfato de levamisole & 2 & 5,1 \\
Sulfóxido de albendazol e & 0 & 0,0 \\
Ivermectina e fosfato de levami sole & 2 & 5,1 \\
Fosfato de levamisole e sul fóxido de albendazole & 1 & 2,6 \\
Ivermectina e sulfóxido de albendazole & 1 & 2,6 \\
Ivermectina, fosfato de levamisole e sulfóxido de albendazole & 1 & 2,6 \\
Sensíveis aos três medicamentos & 4 & 10,3 \\
\hline
\end{tabular}

Ciência Rural, v.38, n.5, ago, 2008. 
Tabela 3 - Número e percentagem de propriedades de criação de bovinos (n=35) com resistência à ivermectina, ao fosfato de levamisole e ao sulfóxido de albendazole por gênero de helmintos.

\begin{tabular}{|c|c|c|c|c|c|c|c|}
\hline \multirow{2}{*}{ Gênero } & \multicolumn{2}{|c|}{ Ivermectina } & \multicolumn{2}{|c|}{ Fosfato de levamisole } & \multicolumn{2}{|c|}{ Sulfóxido de albendazole } & \multirow{2}{*}{ Total } \\
\hline & $\mathrm{N}^{\mathrm{o}}$ & $\%$ & $\mathrm{~N}^{\mathrm{o}}$ & $\%$ & $\mathrm{~N}^{\mathrm{o}}$ & $\%$ & \\
\hline Haemonchus & 5 & 17,2 & - & - & - & - & 5 \\
\hline Cooperia & 6 & 20,7 & 1 & 25,0 & 2 & 100,0 & 9 \\
\hline Ostertagia & 1 & 3,5 & 2 & 50,0 & - & - & 3 \\
\hline Haemonchus e Cooperia & 16 & 55,1 & - & - & - & - & 16 \\
\hline Haemonchus e Ostertagia & 1 & 3,5 & - & - & - & - & 1 \\
\hline Ostertagia e Trichostrongylus & - & - & 1 & 25,0 & - & - & 1 \\
\hline
\end{tabular}

$\mathrm{N}^{\circ}=$ número de propriedades com helmintos resistentes.

Os principais gêneros resistentes à ivermectina foram Haemonchus e Cooperia, constatados em conjunto ou isoladamente, em 27 propriedades. A predominância de resistência desses gêneros está de acordo com os resultados verificados por COSTA et al. (2004) e RANGEL et al. (2005). Foi verificada resistência ao fosfato de levamisole para Ostertagia em três propriedades, sendo uma delas também ao Trichostrongylus, para Cooperia, foi verificada resistência em uma propriedade e, ao sulfóxido de albendazole para Cooperia, em duas propriedades.

\section{CONCLUSÕES}

Com base na literatura e nos resultados do presente trabalho, verificou-se que a situação de resistência dos helmintos de bovinos aos antihelmínticos vem aumentando consideravelmente no país. No Planalto Catarinense, os principais gêneros resistentes à ivermectina são Haemonchus e Cooperia, ao fosfato de levamisole, Ostertagia, Cooperia e Trichostrongylus, e ao sulfóxido de albendazole, Cooperia. Resistência de helmintos à ivermectina foi constatada em um maior número de propriedades.

\section{REFERÊNCIAS}

BORGES, F.A. et al. Resistência de Haemonchus placei, Cooperia punctata e C. spatulata a ivermectina em bovinos do estado de Minas Gerais, Brasil. In: CONGRESSO BRASILEIRO DE PARASITOLOGIA VETERINÁRIA, 13., SIMPÓSIO LATINO-AMERICANO DE RICKSIOSES, 2004, Ouro Preto, SP. Anais... Ouro Preto: CBPV, 2004. p.249.

COLES, G.C. et al. World Association for the Advancement of Veterinary Parasitology (WAAVP) methods for detection of anthelmintic resistence in nematodes of veterinary importance. Veterinary Parasitology, v.44, n.1, p.35-44, 1992.

COSTA, A.J. et al. Avaliação comparativa da ação antihelmíntica e do desenvolvimento ponderal de bezerros tratados com diferentes avermectinas de longa ação. A Hora Veterinária, v.24, n.139, p.31-34, 2004.

ECHEVARRIA, F.A.M.; PINHEIRO, A.C. Eficiência de antihelmínticos em bovinos. In: SEMINÁRIO BRASILEIRO DE PARASITOLOGIA VETERINÁRIA, 11., SEMINÁRIO DE PARASITOLOGIA VETERINÁRIA DOS PAÍSES DO MERCOSUL, 2., 1999, Salvador, BA. Anais... Ilhéus: Universidade Estadual de Santa Cruz, 1999. p.150.

FIEL, C.A. et al. Resistencia antihelmíntica en bovinos: causas, diagnóstico y profilaxis. Veterinaria Argentina, v.18, n.171, p.21-33, 2003.

FIEL C.A. et al. Resistencia antihelmíntica en bovinos. Dos escenarios diferentes como resultado de (1.) El sistema de manejo y (2.) La excesiva frecuencia de tratamientos antiparasitarios. Capturado em 18 de jul. 2006. Online. Disponível na Internet: <http://cnia.inta.gov.ar/ helminto/pdf\%20Resistencia/Fiel.pdf >

GORDON, H. McL.; WHITLOCK, H.V. A new technique for counting nematode eggs in sheep faeces. Journal of Commnwealth Science Industry Organization, v.12, n.1, p.50-52, 1939.

GRIMSHAW, W.T.R. et al. Potential for misinterpretation of the faecal egg count reduction test for levamisole resistant in gastrointestinal nematodes of sheep. Veterinary Parasitology, v.62, p.267-273,1996.

INSTITUTO CEPA/SC. Levantamento agropecuário de Santa Catarina: dados preliminares. Florianópolis, 2005. $255 p$.

JACKSON, F. Anthelmintic resistance - the state of play. British Veterinary Journal, v.149, p.123-138, 1993.

KEITH, R.K. The differentiation of the infective larvae of some common nematodes parasites of cattle. Australian Journal of Zoology, v.1, p.223-235, 1953.

MARTIN, P.J. et al. Detecting benzimidazole resistance with faecal egg count reduction test and in vitro assays. Australian Veterinary Journal, v.45, p.244-246, 1989.

PAIVA, F. et al. Resistência a ivermectina constatada em Haemonchus placei e Cooperia punctata em bovinos. A Hora Veterinária, v.20, n.120, p.29-32, 2001. 
PINHEIRO, A.C.; ECHEVARRIA, F.A.M. Susceptibilidade de Haemonchus spp em bovinos ao tratamento anti-helmíntico com albendazole e oxfendazole. Pesquisa Veterinária Brasileira, v.10, n.1/2, p.19-21, 1990.

PRICHARD, R.K. Anthelmintic resistance. Veterinary Parasitology, v.54, p.259-268, 1994.

RAMOS, C.I. et al. Sistemas de tratamentos antihelmínticos no Planalto Catarinense. Florianópolis: Empasc, 1984. 23p. (Boletim Técnico, 250).

RANGEL, V.B. et al. Resistência de Cooperia spp e Haemonchus spp as avermectinas em bovinos de corte. Arquivo Brasileiro de Medicina Veterinária e Zootecnia, v.57, n.2. p.186190, 2005.
ROBERTS, F.H.S.; O’SULLIVAN, P.J. Methods for eggs-counts and larval cultures for strongyles infesting the gastrointestinal tract of cattle. Australian Journal of Agricultural Research, v.1, n.1, p.99-102, 1950.

SOUZA et al. Resistência de helmintos gastrintestinais de bovinos a anti-helmínticos no estado de Santa Catarina. In: CONGRESSO LATINO-AMERICANO DE PARASITOLOGIA, 15, 2001, São Paulo, SP. Suplemento Científico... São Paulo: SBPC, SBP, SBC, 2001. p.233.

SOUZA, A.P. et al. Controle estratégico do Boophilus microplus com o uso de Doramectin e o efeito sobre a dinâmica populacional de Dermatobia hominis e helmintos gastrintestinais. In: JORNADA DE PESQUISA DA UDESC, 3. 1994, Florianópolis, SC. Anais... Florianópolis: UDESC, 1994. p.98. 